Article

\title{
Asymptotic Behavior of Solutions of the Third Order Nonlinear Mixed Type Neutral Differential Equations
}

\author{
Osama Moaaz ${ }^{1,+}$ (1) Dimplekumar Chalishajar ${ }^{2, *,+}$ and Omar Bazighifan ${ }^{3,4,+}$ (i) \\ 1 Department of Mathematics, Faculty of Science, Mansoura University, Mansoura 35516, Egypt; \\ o_moaaz@mans.edu.eg \\ 2 Department of Applied Mathematics, Virginia Military Institute (VMI) 435 Mallory Hall, Lexington, \\ VA 24450, USA \\ 3 Department of Mathematics, Faculty of Science, Hadhramout University, Hadhramout 50512, Yemen; \\ o.bazighifan@gmail.com \\ 4 Department of Mathematics, Faculty of Education, Seiyun University, Hadhramout 50512, Yemen \\ * Correspondence: chalishajardn@vmi.edu \\ + These authors contributed equally to this work.
}

Received: 29 January 2020; Accepted: 20 March 2020; Published: 1 April 2020

Abstract: The objective of our paper is to study asymptotic properties of the class of third order neutral differential equations with advanced and delayed arguments. Our results supplement and improve some known results obtained in the literature. An illustrative example is provided.

Keywords: oscillation; third order; mixed neutral differential equations

\section{Introduction}

Equations with neutral terms are of particular significance, as they arise in many applications including systems of control, electrodynamics, mixing liquids, neutron transportation, networks and population models; see [1].

Asymptotic properties of solutions of second/third order differential equations have been subject to intensive research in the literature. This problem for differential equations with respective delays has received a great deal of attention in the last years; see for examples, [2-21].

This paper deals with the oscillation and asymptotic behavior of solutions of the class of third-order, nonlinear, mixed-type, neutral differential equations

$$
\left(r(t)\left(z^{\prime \prime}(t)\right)^{\alpha}\right)^{\prime}+q_{1}(t) f_{1}\left(x\left(\sigma_{1}(t)\right)\right)+q_{2}(t) f_{2}\left(x\left(\sigma_{2}(t)\right)\right)=0,
$$

where

$$
z(t)=x(t)+p_{1}(t) x\left(\tau_{1}(t)\right)+p_{2}(t) x\left(\tau_{2}(t)\right)
$$

and we will assume the following assumptions hold:

$\left(\mathrm{M}_{1}\right) \quad r \in C\left(\left[t_{0}, \infty\right),(0, \infty)\right), \int_{t_{0}}^{\infty} r^{-1 / \alpha}(s) \mathrm{d} s=\infty$ and $\alpha$ is a ratio of odd positive integers;

$\left(\mathrm{M}_{2}\right) \quad p_{i} \in C\left(\left[t_{0}, \infty\right),\left[0, c_{i}\right]\right)$ where $c_{i}$ are constants for $i=1,2$ and $c_{1}+c_{2}<1$;

$\left(\mathrm{M}_{3}\right) \quad \tau_{i}, \sigma_{i} \in C\left(\left[t_{0}, \infty\right), \mathbb{R}\right), \tau_{1}(t)<t, \sigma_{1}(t)<t, \tau_{2}(t)>t, \sigma_{2}(t)>t, \sigma_{i}\left(\tau_{i}(t)\right)=\tau_{i}\left(\sigma_{i}(t)\right)$ and $\lim _{t \rightarrow \infty} \tau_{i}(t)=\lim _{t \rightarrow \infty} \sigma_{i}(t)=\infty$ for $i=1,2$;

$\left(\mathrm{M}_{4}\right) \quad q_{i} \in C\left(\left[t_{0}, \infty\right),(0, \infty)\right)$ for $i=1,2$; 
$\left(\mathrm{M}_{5}\right) \quad f_{1}, f_{2} \in C(\mathbb{R}, \mathbb{R}), f_{1}(x) / x^{\beta} \geq k_{1}>0$ and $f_{2}(x) / x^{\gamma} \geq k_{2}$ for $x \neq 0$ where $\beta$ and $\gamma$ are ratios of odd positive integers.

By a solution of Equation (1), we mean a non-trivial real function $x \in C\left(\left[t_{x}, \infty\right)\right), t_{x} \geq t_{0}$, with $z(t), z^{\prime}(t)$ and $r_{1}(t)\left(z^{\prime \prime}(t)\right)^{\alpha}$ being continuously differentiable for all $t \in\left[t_{x}, \infty\right)$, and satisfying (1) on $\left[t_{x}, \infty\right)$. A solution of Equation (1) is called oscillatory if it has arbitrary large zeros; otherwise it is called nonoscillatory. Equation (1) is said to be oscillatory if all its solutions are oscillatory.

Han et al. in [22] studied the asymptotic properties of the solutions of equation

$$
\left(r(t)\left(z^{\prime \prime}(t)\right)\right)^{\prime}+q_{1}(t) x\left(\sigma_{1}(t)\right)+q_{2}(t) x\left(\sigma_{2}(t)\right)=0,
$$

where $z(t)=x(t)+p_{1}(t) x\left(\tau_{1}(t)\right)+p_{2}(t) x\left(\tau_{2}(t)\right)$.

Baculikova and Dzurina [5] studied the oscillation of the third-order equation

$$
\left(r(t)\left(x^{\prime}(t)\right)^{\alpha}\right)^{\prime \prime}+q(t) f(x(\tau(t)))+p(t) h(x(\sigma(t)))=0,
$$

where $\tau(t) \leq t$ and $\sigma(t) \geq t$.

Thandapani and Rama [23] established some oscillation theorems for equation

$$
\left(r(t)\left(z^{\prime \prime}(t)\right)\right)^{\prime}+q_{1}(t) x^{\alpha}\left(\sigma_{1}(t)\right)+q_{2}(t) x^{\beta}\left(\sigma_{2}(t)\right)=0,
$$

where $z(t)=x(t)+p_{1}(t) x\left(\tau_{1}(t)\right)+p_{2}(t) x\left(\tau_{2}(t)\right)$, and the authors used the Recati technique.

The aim of this paper is to discuss the asymptotic behavior of solutions of a class of third-order, nonlinear, mixed-type, neutral differential equations. We established sufficient conditions to ensure that the solution of Equation (1) is oscillatory or tended to zero. The results of this study basically generalize and improve the previous results. An illustrative example is provided.

\section{Auxiliary Lemmas}

In order to prove our results, we shall need the next auxiliary lemmas.

Lemma 1. Assume that $f(y)=U y-V y^{\frac{\eta+1}{\eta}}$, where $U$ and $V$ are constants, $V>0$ and $\eta$ is a quotient of odd positive integers. Then $f$ imposes its maximum value on $\mathbb{R}$ at $y^{*}=\left(\frac{U \eta}{V(\eta+1)}\right)^{\eta}$ and

$$
\max _{y \in \mathbb{R}} f=f\left(y^{*}\right)=\frac{\eta^{\eta}}{(\eta+1)^{\eta+1}} U^{\eta+1} V^{-\eta} .
$$

Lemma 2 ([24]). Assume that $A \geq 0$ and $B \geq 0$. If $\delta>1$, then

$$
(A+B)^{\delta} \leq 2^{\delta-1}\left(A^{\delta}+B^{\delta}\right)
$$

Moreover, if $0<\delta<1$, then $(A+B)^{\delta} \leq\left(A^{\delta}+B^{\delta}\right)$.

Lemma 3 ([17]). If the function $y$ satisfies $y^{(i)}>0, i=0,1, \ldots, n$, and $y^{(n+1)}<0$, then

$$
\frac{y(t)}{t^{n} / n !} \geq \frac{y^{\prime}(t)}{t^{n-1} /(n-1) !}
$$


Lemma 4 ([23]). Assume that $u(t)>0, u^{\prime}(t)>0, u^{\prime \prime}(t)>0$ and $u^{\prime \prime \prime}(t)<0$ on $(T, \infty)$. Then,

$$
\frac{u(t)}{u^{\prime}(t)} \geq \frac{t-T}{2} \geq \frac{\mu t}{2}
$$

for $t \geq T$ and some $\mu \in(0,1)$.

Lemma 5. Let $x$ be a positive solution of Equation (1). Then $z$ has only one of the following two properties eventually:

(i) $z(t)>0, z^{\prime}(t)>0$ and $z^{\prime \prime}(t)>0$;

(ii) $z(t)>0, z^{\prime}(t)<0$ and $z^{\prime \prime}(t)>0$.

Proof. The proof is similar to that of Lemma 2.1 of [10] and hence the details are omitted.

Lemma 6. Let $x$ be a positive solution of Equation (1), and $z$ has the property (ii). If $\beta=\gamma$ and

$$
\left.\int_{t_{0}}^{\infty} \int_{v}^{\infty}\left(\frac{1}{r(u)} \int_{u}^{\infty}\left(k_{1} q_{1}(s)+k_{2} q_{2}(s)\right)\right) \mathrm{d} s\right)^{1 / \alpha} \mathrm{d} u \mathrm{~d} v=\infty
$$

then the solution $x$ of Equation (1) converges to zero as $t \rightarrow \infty$.

Proof. Let $x$ be a positive solution of Equation (1). Since $z$ satisfies the property (ii), we get $\lim _{t \rightarrow \infty} z(t)=$ $\delta \geq 0$. Next, we will prove that $\delta=0$. Suppose that $\delta>0$, then we have for all $\varepsilon>0$ and $t$ enough large $\delta<z(t)<\delta+\varepsilon$. By choosing $\varepsilon<\frac{1-c_{1}-c_{2}}{c_{1}+c_{2}} \delta$, we obtain

$$
\begin{aligned}
x(t) & =z(t)-p_{1}(t) x\left(\tau_{1}(t)\right)-p_{2}(t) x\left(\tau_{2}(t)\right) \\
& >\delta-\left(c_{1}+c_{2}\right) z\left(\tau_{1}(t)\right) \\
& >\delta-\left(c_{1}+c_{2}\right)(\delta+\varepsilon) \\
& >L(\delta+\varepsilon)>L z(t)
\end{aligned}
$$

where $L=\frac{\delta-\left(c_{1}+c_{2}\right)(\delta+\varepsilon)}{\delta+\varepsilon}>0$. Thus, from (1) and $\left(\mathrm{M}_{5}\right)$, we have

$$
\begin{aligned}
0 & \geq\left(r(t)\left(z^{\prime \prime}(t)\right)^{\alpha}\right)^{\prime}+k_{1} q_{1}(t) x^{\beta}\left(\sigma_{1}(t)\right)+k_{2} q_{2}(t) x^{\beta}\left(\sigma_{2}(t)\right) \\
& \geq\left(r(t)\left(z^{\prime \prime}(t)\right)^{\alpha}\right)^{\prime}+L^{\beta}\left(k_{1} q_{1}(t)+k_{2} q_{2}(t)\right) z^{\beta}\left(\sigma_{2}(t)\right)
\end{aligned}
$$

and so,

$$
\left(r(t)\left(z^{\prime \prime}(t)\right)^{\alpha}\right)^{\prime} \leq-L^{\beta} \delta^{\beta}\left(k_{1} q_{1}(t)+k_{2} q_{2}(t)\right) .
$$

By integrating this inequality two times from $t$ to $\infty$, we get

$$
\left.-z^{\prime}(t)>L^{\beta / \alpha} \delta^{\beta / \alpha} \int_{t}^{\infty}\left(\frac{1}{r(u)} \int_{u}^{\infty}\left(k_{1} q_{1}(s)+k_{2} q_{2}(s)\right)\right) \mathrm{d} s\right)^{1 / \alpha} \mathrm{d} u .
$$

Integrating the last inequality from $t_{1}$ to $\infty$, we have

$$
\left.z\left(t_{1}\right)>L^{\beta / \alpha} \delta^{\beta / \alpha} \int_{t_{1}}^{\infty} \int_{v}^{\infty}\left(\frac{1}{r(u)} \int_{u}^{\infty}\left(k_{1} q_{1}(s)+k_{2} q_{2}(s)\right)\right) \mathrm{d} s\right)^{1 / \alpha} \mathrm{d} u \mathrm{~d} v .
$$


Thus, we are led to a contradiction with (3). Then, $\lim _{t \rightarrow \infty} z(t)=0$; moreover, the fact that $x(t) \leq z(t)$ implies $\lim _{t \rightarrow \infty} x(t)=0$.

\section{Main Results}

In this section, we will establish new oscillation criteria for solutions of the Equation (1). For the sake of convenience, we insert the next notation:

$$
\begin{gathered}
R_{u}(t):=\int_{u}^{t} \frac{1}{r^{1 / \alpha}(s)} \mathrm{d} s, \\
R_{u}^{*}(t):=\min _{t \geq t_{0}}\left\{R_{u}(t), R_{u}\left(\tau_{1}(t)\right)\right\}
\end{gathered}
$$

and

$$
q_{i}^{*}(t):=\min _{t \geq t_{0}}\left\{q_{i}(t), q_{i}\left(\tau_{1}(t)\right), q_{i}\left(\tau_{2}(t)\right)\right\}, i=1,2 .
$$

Theorem 1. Assume that $\left(M_{1}\right)-\left(M_{5}\right)$ and (3) hold. Let $\beta=\gamma \geq \alpha, \sigma_{1}(t) \leq \tau_{1}(t)$ and $\sigma_{1}^{\prime}(t)>0$. If there exists a positive function $\rho \in C^{1}\left(\left[t_{0}, \infty\right)\right)$ such that

$$
\limsup _{t \rightarrow \infty} \int_{t_{0}}^{t}\left(\Theta_{1}(s)-\left(1+c_{1}^{\beta}+\frac{c_{2}^{\beta}}{2^{\beta-1}}\right) \frac{1}{(\alpha+1)^{\alpha+1}} \frac{\left(\rho_{+}^{\prime}(s)\right)^{\alpha+1} r\left(\sigma_{1}(s)\right)}{\left(\rho(s) \sigma_{1}^{\prime}(s)\right)^{\alpha}}\right) \mathrm{d} s=\infty,
$$

where $\rho_{+}^{\prime}(s)=\max \left\{\rho^{\prime}(s), 0\right\}$ and

$$
\Theta_{1}(t)=\frac{\mu^{\alpha} v^{\beta-\alpha}}{2^{2 \beta+\alpha-2}} \rho(t) \sigma_{1}^{\beta}(t)\left(k_{1} q_{1}^{*}(t)+k_{2} q_{2}^{*}(t)\right),
$$

then every solution of equation (1) either oscillates or tends to zero as $t \rightarrow \infty$.

Proof. Let $x$ be non-oscillatory solution of Equation (1). Without loss of generality, we assume that $x(t)>0$; then there exists a $t_{1} \geq t_{0}$ such that $x(t)>0, x\left(\tau_{i}(t)\right)>0$ and $x\left(\sigma_{i}(t)\right)>0$ for $t \geq t_{1}$ and $i=1$, 2. From Lemma 5 , we have that $z$ has the property (i) or the property (ii). From Lemma 6 , if $z(t)$ has the property (ii), then we obtain $\lim _{t \rightarrow \infty} x(t)=0$. Next, let $z$ have the property (i). Using (1) and $\left(\mathrm{M}_{5}\right)$, we obtain

$$
\left(r(t)\left(z^{\prime \prime}(t)\right)^{\alpha}\right)^{\prime}+k_{1} q_{1}(t) x^{\beta}\left(\sigma_{1}(t)\right)+k_{2} q_{2}(t) x^{\beta}\left(\sigma_{2}(t)\right) \leq 0 .
$$

Thus, we get

$$
\begin{aligned}
0 \geq & \left(r(t)\left(z^{\prime \prime}(t)\right)^{\alpha}\right)^{\prime}+k_{1} q_{1}(t) x^{\beta}\left(\sigma_{1}(t)\right)+k_{2} q_{2}(t) x^{\beta}\left(\sigma_{2}(t)\right) \\
& +c_{1}^{\beta}\left[\left(r\left(\tau_{1}(t)\right)\left(z^{\prime \prime}\left(\tau_{1}(t)\right)\right)^{\alpha}\right)^{\prime}+k_{1} q_{1}\left(\tau_{1}(t)\right) x^{\beta}\left(\sigma_{1}\left(\tau_{1}(t)\right)\right)\right. \\
& \left.+k_{2} q_{2}\left(\tau_{1}(t)\right) x^{\beta}\left(\sigma_{2}\left(\tau_{1}(t)\right)\right)\right]+\frac{c_{2}^{\beta}}{2^{\beta-1}}\left[\left(r\left(\tau_{2}(t)\right)\left(z^{\prime \prime}\left(\tau_{2}(t)\right)\right)^{\alpha}\right)^{\prime}\right. \\
& \left.+k_{1} q_{1}\left(\tau_{2}(t)\right) x^{\beta}\left(\sigma_{1}\left(\tau_{2}(t)\right)\right)+k_{2} q_{2}\left(\tau_{2}(t)\right) x^{\beta}\left(\sigma_{2}\left(\tau_{2}(t)\right)\right)\right] .
\end{aligned}
$$


That is

$$
\begin{aligned}
(r(t) & \left.\left(z^{\prime \prime}(t)\right)^{\alpha}\right)^{\prime}+c_{1}^{\beta}\left(r\left(\tau_{1}(t)\right)\left(z^{\prime \prime}\left(\tau_{1}(t)\right)\right)^{\alpha}\right)^{\prime}+\frac{c_{2}^{\beta}}{2^{\beta-1}}\left(r\left(\tau_{2}(t)\right)\left(z^{\prime \prime}\left(\tau_{2}(t)\right)\right)^{\alpha}\right)^{\prime} \\
& +k_{1} q_{1}^{*}(t)\left(x^{\beta}\left(\sigma_{1}(t)\right)+c_{1}^{\beta} x^{\beta}\left(\sigma_{1}\left(\tau_{1}(t)\right)\right)+\frac{c_{2}^{\beta}}{2^{\beta-1}} x^{\beta}\left(\sigma_{1}\left(\tau_{2}(t)\right)\right)\right) \\
& +k_{2} q_{2}^{*}(t)\left(x^{\beta}\left(\sigma_{2}(t)\right)+c_{1}^{\beta} x^{\beta}\left(\sigma_{2}\left(\tau_{1}(t)\right)\right)+\frac{c_{2}^{\beta}}{2^{\beta-1}} x^{\beta}\left(\sigma_{2}\left(\tau_{2}(t)\right)\right)\right) \leq 0 .
\end{aligned}
$$

From Lemma 2, we obtain

$$
\begin{aligned}
z^{\beta}(t) & \leq\left(x(t)+c_{1}(t) x\left(\tau_{1}(t)\right)+c_{2}(t) x\left(\tau_{2}(t)\right)\right)^{\beta} \\
& \leq 4^{\beta-1}\left(x^{\beta}(t)+c_{1}^{\beta} x^{\beta}\left(\tau_{1}(t)\right)+\frac{c_{2}^{\beta}}{2^{\beta-1}} x^{\beta}\left(\tau_{2}(t)\right)\right)
\end{aligned}
$$

which with (5) gives

$$
\begin{gathered}
\left(r(t)\left(z^{\prime \prime}(t)\right)^{\alpha}\right)^{\prime}+c_{1}^{\beta}\left(r\left(\tau_{1}(t)\right)\left(z^{\prime \prime}\left(\tau_{1}(t)\right)\right)^{\alpha}\right)^{\prime}+\frac{c_{2}^{\beta}}{2^{\beta-1}}\left(r\left(\tau_{2}(t)\right)\left(z^{\prime \prime}\left(\tau_{2}(t)\right)\right)^{\alpha}\right)^{\prime} \\
+\frac{k_{1}}{4^{\beta-1}} q_{1}^{*}(t) z^{\beta}\left(\sigma_{1}(t)\right)+\frac{k_{2}}{4^{\beta-1}} q_{2}^{*}(t) z^{\beta}\left(\sigma_{2}(t)\right) \leq 0 .
\end{gathered}
$$

This implies that

$$
\begin{gathered}
\left(r(t)\left(z^{\prime \prime}(t)\right)^{\alpha}\right)^{\prime}+c_{1}^{\beta}\left(r\left(\tau_{1}(t)\right)\left(z^{\prime \prime}\left(\tau_{1}(t)\right)\right)^{\alpha}\right)^{\prime}+\frac{c_{2}^{\beta}}{2^{\beta-1}}\left(r\left(\tau_{2}(t)\right)\left(z^{\prime \prime}\left(\tau_{2}(t)\right)\right)^{\alpha}\right)^{\prime} \\
+\frac{1}{4^{\beta-1}}\left(k_{1} q_{1}^{*}(t)+k_{2} q_{2}^{*}(t)\right) z^{\beta}\left(\sigma_{1}(t)\right) \leq 0 .
\end{gathered}
$$

Now, we define

$$
\omega_{1}(t)=\rho(t) \frac{r(t)\left(z^{\prime \prime}(t)\right)^{\alpha}}{\left(z^{\prime}\left(\sigma_{1}(t)\right)\right)^{\alpha}} .
$$

Then $\omega_{1}(t)>0$. By differentiating, we get

$$
\omega_{1}^{\prime}(t)=\frac{\rho^{\prime}(t)}{\rho(t)} \omega_{1}(t)+\rho(t) \frac{\left(r(t)\left(z^{\prime \prime}(t)\right)^{\alpha}\right)^{\prime}}{\left(z^{\prime}\left(\sigma_{1}(t)\right)\right)^{\alpha}}-\alpha \rho(t) \frac{r(t)\left(z^{\prime \prime}(t)\right)^{\alpha}}{\left(z^{\prime}\left(\sigma_{1}(t)\right)\right)^{\alpha+1}} z^{\prime \prime}\left(\sigma_{1}(t)\right) \sigma_{1}^{\prime}(t) .
$$

Since $\left(r(t)\left(z^{\prime \prime}(t)\right)^{\alpha}\right)^{\prime}<0$ and $\sigma_{1}(t)<t$, we obtain

$$
r(t)\left(z^{\prime \prime}(t)\right)^{\alpha} \leq r\left(\sigma_{1}(t)\right)\left(z^{\prime \prime}\left(\sigma_{1}(t)\right)\right)^{\alpha},
$$

and hence

$$
\omega_{1}^{\prime}(t) \leq \frac{\rho_{+}^{\prime}(t)}{\rho(t)} \omega_{1}(t)-\alpha \frac{\sigma_{1}^{\prime}(t)}{\rho^{1 / \alpha}(t) r^{1 / \alpha}\left(\sigma_{1}(t)\right)} \omega_{1}^{\frac{\alpha+1}{\alpha}}(t)+\rho(t) \frac{\left(r(t)\left(z^{\prime \prime}(t)\right)^{\alpha}\right)^{\prime}}{\left(z^{\prime}\left(\sigma_{1}(t)\right)\right)^{\alpha}}
$$


Using Lemma 1 with

$$
\eta=\alpha, U=\frac{\rho_{+}^{\prime}(t)}{\rho(t)}, V=\alpha \frac{\sigma_{1}^{\prime}(t)}{\rho^{1 / \alpha}(t) r^{1 / \alpha}\left(\sigma_{1}(t)\right)} \text { and } y=\omega_{1}
$$

we obtain

$$
\omega_{1}^{\prime}(t) \leq \rho(t) \frac{\left(r(t)\left(z^{\prime \prime}(t)\right)^{\alpha}\right)^{\prime}}{\left(z^{\prime}\left(\sigma_{1}(t)\right)\right)^{\alpha}}+\frac{1}{(\alpha+1)^{\alpha+1}} \frac{\left(\rho_{+}^{\prime}(t)\right)^{\alpha+1} r\left(\sigma_{1}(t)\right)}{\left(\rho(t) \sigma_{1}^{\prime}(t)\right)^{\alpha}} .
$$

Further, we define the function

$$
\omega_{2}(t)=\rho(t) \frac{r\left(\tau_{1}(t)\right)\left(z^{\prime \prime}\left(\tau_{1}(t)\right)\right)^{\alpha}}{\left(z^{\prime}\left(\sigma_{1}(t)\right)\right)^{\alpha}} .
$$

Then $\omega_{2}(t)>0$. By differentiating $\omega_{2}$ and using $\sigma_{1}(t) \leq \tau_{1}(t)$, we find

$$
\omega_{2}^{\prime}(t) \leq \frac{\rho^{\prime}(t)}{\rho(t)} \omega_{2}(t)+\rho(t) \frac{\left(r\left(\tau_{1}(t)\right)\left(z^{\prime \prime}\left(\tau_{1}(t)\right)\right)^{\alpha}\right)^{\prime}}{\left(z^{\prime}\left(\sigma_{1}(t)\right)\right)^{\alpha}}-\alpha \frac{\sigma_{1}^{\prime}(t)}{\rho^{1 / \alpha}(t) r^{1 / \alpha}\left(\sigma_{1}(t)\right)} \omega_{2}^{\frac{\alpha+1}{\alpha}}(t) .
$$

Using Lemma 1, we obtain

$$
\omega_{2}^{\prime}(t) \leq \rho(t) \frac{\left(r\left(\tau_{1}(t)\right)\left(z^{\prime \prime}\left(\tau_{1}(t)\right)\right)^{\alpha}\right)^{\prime}}{\left(z^{\prime}\left(\sigma_{1}(t)\right)\right)^{\alpha}}+\frac{1}{(\alpha+1)^{\alpha+1}} \frac{\left(\rho_{+}^{\prime}(t)\right)^{\alpha+1} r\left(\sigma_{1}(t)\right)}{\left(\rho(t) \sigma_{1}^{\prime}(t)\right)^{\alpha}} .
$$

Next, we define another function

$$
\omega_{3}(t)=\rho(t) \frac{r\left(\tau_{2}(t)\right)\left(z^{\prime \prime}\left(\tau_{2}(t)\right)\right)^{\alpha}}{\left(z^{\prime}\left(\sigma_{1}(t)\right)\right)^{\alpha}} .
$$

Thus $\omega_{3}(t)>0$. By differentiating, and similar to (9) we have

$$
\omega_{3}^{\prime}(t) \leq \rho(t) \frac{\left(r\left(\tau_{2}(t)\right)\left(z^{\prime \prime}\left(\tau_{2}(t)\right)\right)^{\alpha}\right)^{\prime}}{\left(z^{\prime}\left(\sigma_{1}(t)\right)\right)^{\alpha}}+\frac{1}{(\alpha+1)^{\alpha+1}} \frac{\left(\rho_{+}^{\prime}(t)\right)^{\alpha+1} r\left(\sigma_{1}(t)\right)}{\left(\rho(t) \sigma_{1}^{\prime}(t)\right)^{\alpha}} .
$$

From (8)-(10), we get

$$
\begin{aligned}
\omega_{1}^{\prime}(t)+ & c_{1}^{\beta} \omega_{2}^{\prime}(t)+\frac{c_{2}^{\beta}}{2^{\beta-1}} \omega_{3}^{\prime}(t) \leq \frac{\rho(t)}{\left(z^{\prime}\left(\sigma_{1}(t)\right)\right)^{\alpha}}\left(\left(r(t)\left(z^{\prime \prime}(t)\right)^{\alpha}\right)^{\prime}+\right. \\
& \left.+c_{1}^{\beta}\left(r\left(\tau_{1}(t)\right)\left(z^{\prime \prime}\left(\tau_{1}(t)\right)\right)^{\alpha}\right)^{\prime}+\frac{c_{2}^{\beta}}{2^{\beta-1}}\left(r\left(\tau_{2}(t)\right)\left(z^{\prime \prime}\left(\tau_{2}(t)\right)\right)^{\alpha}\right)^{\prime}\right) \\
& +\left(1+c_{1}^{\beta}+\frac{c_{2}^{\beta}}{2^{\beta-1}}\right) \frac{1}{(\alpha+1)^{\alpha+1}} \frac{\left(\rho_{+}^{\prime}(t)\right)^{\alpha+1} r\left(\sigma_{1}(t)\right)}{\left(\rho(t) \sigma_{1}^{\prime}(t)\right)^{\alpha}},
\end{aligned}
$$

which with (7) gives

$$
\begin{gathered}
\omega_{1}^{\prime}(t)+c_{1}^{\beta} \omega_{2}^{\prime}(t)+\frac{c_{2}^{\beta}}{2^{\beta-1}} \omega_{3}^{\prime}(t) \leq-\frac{\rho(t)}{4^{\beta-1}}\left(k_{1} q_{1}^{*}(t)+k_{2} q_{2}^{*}(t)\right) \frac{z^{\beta}\left(\sigma_{1}(t)\right)}{\left(z^{\prime}\left(\sigma_{1}(t)\right)\right)^{\alpha}} \\
+\left(1+c_{1}^{\beta}+\frac{c_{2}^{\beta}}{2^{\beta-1}}\right) \frac{1}{(\alpha+1)^{\alpha+1}} \frac{\left(\rho_{+}^{\prime}(t)\right)^{\alpha+1} r\left(\sigma_{1}(t)\right)}{\left(\rho(t) \sigma_{1}^{\prime}(t)\right)^{\alpha}} .
\end{gathered}
$$


Using Lemma 4, we have, for some $\mu \in(0,1)$,

$$
\frac{z\left(\sigma_{1}(t)\right)}{z^{\prime}\left(\sigma_{1}(t)\right)} \geq \frac{\mu}{2} \sigma_{1}(t)
$$

From property (i), we get

$$
\begin{aligned}
z(t) & =z\left(t_{1}\right)+\int_{t_{1}}^{t} z^{\prime}(s) d s \\
& \geq\left(t-t_{1}\right) z^{\prime}\left(t_{1}\right) \geq \frac{v}{2} t
\end{aligned}
$$

for some $v>0$ and for $t$ enough large. Therefore, for some $\mu \in(0,1)$ and $v>0$, we find

$$
\frac{z^{\beta}\left(\sigma_{1}(t)\right)}{\left(z^{\prime}\left(\sigma_{1}(t)\right)\right)^{\alpha}} \geq \frac{\mu^{\alpha} v^{\beta-\alpha}}{2^{\alpha}} \sigma_{1}^{\beta}(t) .
$$

Combining the last inequality with (11), we obtain

$$
\begin{aligned}
\omega_{1}^{\prime}(t)+c_{1}^{\beta} \omega_{2}^{\prime}(t) & +\frac{c_{2}^{\beta}}{2^{\beta-1}} \omega_{3}^{\prime}(t) \leq-\Theta(t) \\
& +\left(1+c_{1}^{\beta}+\frac{c_{2}^{\beta}}{2^{\beta-1}}\right) \frac{1}{(\alpha+1)^{\alpha+1}} \frac{\left(\rho_{+}^{\prime}(t)\right)^{\alpha+1} r\left(\sigma_{1}(t)\right)}{\left(\rho(t) \sigma_{1}^{\prime}(t)\right)^{\alpha}} .
\end{aligned}
$$

Integrating the above inequality from $t_{1}$ to $t$, we have

$$
\begin{gathered}
\int_{t_{1}}^{t}\left(\Theta(s)-\left(1+c_{1}^{\beta}+\frac{c_{2}^{\beta}}{2^{\beta-1}}\right) \frac{1}{(\alpha+1)^{\alpha+1}} \frac{\left(\rho_{+}^{\prime}(s)\right)^{\alpha+1} r\left(\sigma_{1}(s)\right)}{\left(\rho(s) \sigma_{1}^{\prime}(s)\right)^{\alpha}}\right) d s \\
\leq \omega_{1}\left(t_{1}\right)+c_{1}^{\beta} \omega_{2}\left(t_{1}\right)+\frac{c_{2}^{\beta}}{2^{\beta-1}} \omega_{3}\left(t_{1}\right) .
\end{gathered}
$$

Taking the superior limit as $t \rightarrow \infty$, we get a contradiction with (4). The proof is complete.

Remark 1. In the Theorem 1, if $\sigma_{1}(t) \geq \tau_{1}(t)$ and $\tau_{1}^{\prime}(t)>0$, then the assumption (4) is replaced by

$$
\limsup _{t \rightarrow \infty} \int_{t_{0}}^{t}\left(\Theta_{1}(s)-\left(1+c_{1}^{\beta}+\frac{c_{2}^{\beta}}{2^{\beta-1}}\right) \frac{1}{(\alpha+1)^{\alpha+1}} \frac{\left(\rho_{+}^{\prime}(s)\right)^{\alpha+1} r\left(\tau_{1}(s)\right)}{\left(\rho(s) \tau_{1}^{\prime}(s)\right)^{\alpha}}\right) d s=\infty .
$$

Theorem 2. Assume that $\left(M_{1}\right)-\left(M_{5}\right)$ and (3) hold. Let $\beta=\gamma \geq \alpha$ and $r^{\prime}(t)>0$. If there exists a positive function $\rho \in C^{1}\left(\left[t_{0}, \infty\right)\right)$ such that

$$
\limsup _{t \rightarrow \infty} \int_{t_{0}}^{t}\left(\Theta_{2}(s)-\left(1+c_{1}^{\beta}+\frac{c_{2}^{\beta}}{2^{\beta-1}}\right) \frac{1}{(\alpha+1)^{\alpha+1}} \frac{\left(\rho_{+}^{\prime}(t)\right)^{\alpha+1}}{\left(\rho(t) R_{t_{0}}^{*}(t)\right)^{\alpha}}\right) d s=\infty,
$$

where

$$
\Theta_{2}(t)=\frac{v^{\beta-\alpha}}{2^{3 \beta-\alpha-2} t^{2 \alpha}} \rho(t) \sigma_{1}^{\beta+\alpha}(t)\left(k_{1} q_{1}^{*}(t)+k_{2} q_{2}^{*}(t)\right)
$$


then every solution of Equation (1) either oscillates or tends to zero as $t \rightarrow \infty$.

Proof. Proceeding as in the proof of Theorem 1, we have that (7) holds. Since $\left(r(t)\left(z^{\prime \prime}(t)\right)^{\alpha}\right)^{\prime}<0$, we obtain

$$
\begin{aligned}
z^{\prime}(t) & =z^{\prime}\left(t_{1}\right)+\int_{t_{1}}^{t} \frac{\left[r(s)\left(z^{\prime \prime}(s)\right)^{\alpha}\right]^{1 / \alpha}}{r^{1 / \alpha}(s)} d s \\
& \geq\left[r(t)\left(z^{\prime \prime}(t)\right)^{\alpha}\right]^{1 / \alpha} R_{t_{1}}(t) .
\end{aligned}
$$

Now, we define

$$
\omega_{1}(t)=\rho(t) \frac{r(t)\left(z^{\prime \prime}(t)\right)^{\alpha}}{z^{\alpha}(t)} .
$$

Then $\omega_{1}(t)>0$. By differentiating $\omega_{1}$ and using (14), we get

$$
\omega_{1}^{\prime}(t) \leq \frac{\rho_{+}^{\prime}(t)}{\rho(t)} \omega_{1}(t)-\alpha \frac{R_{t_{1}}(t)}{\rho^{1 / \alpha}(t)} \omega_{1}^{\frac{\alpha+1}{\alpha}}(t)+\rho(t) \frac{\left(r(t)\left(z^{\prime \prime}(t)\right)^{\alpha}\right)^{\prime}}{z^{\alpha}(t)} .
$$

Using Lemma 1 with $\eta=\alpha, U=\frac{\rho_{+}^{\prime}(t)}{\rho(t)}, V=\alpha \frac{R_{t_{1}}(t)}{\rho^{1 / \alpha}(t)}$ and $y=\omega_{1}$, we obtain

$$
\omega_{1}^{\prime}(t) \leq \rho(t) \frac{\left(r(t)\left(z^{\prime \prime}(t)\right)^{\alpha}\right)^{\prime}}{z^{\alpha}(t)}+\frac{1}{(\alpha+1)^{\alpha+1}} \frac{\left(\rho_{+}^{\prime}(t)\right)^{\alpha+1}}{\left(\rho(t) R_{t_{1}}(t)\right)^{\alpha}}
$$

Next, we define a function

$$
\omega_{2}(t)=\rho(t) \frac{r\left(\tau_{1}(t)\right)\left(z^{\prime \prime}\left(\tau_{1}(t)\right)\right)^{\alpha}}{z^{\alpha}(t)} .
$$

Then $\omega_{2}(t)>0$. Since $z^{\prime \prime}(t)>0$ and $\tau_{1}(t)<t$, we obtain $z^{\prime}(t)>z^{\prime}\left(\tau_{1}(t)\right)$. Hence, from (14), we find

$$
z^{\prime}(t)>\left[r\left(\tau_{1}(t)\right)\left(z^{\prime \prime}\left(\tau_{1}(t)\right)\right)^{\alpha}\right]^{1 / \alpha} R_{t_{1}}\left(\tau_{1}(t)\right) .
$$

for $t \geq t_{2} \geq t_{1}$. By differentiating (16) and using (17), we get

$$
\omega_{2}^{\prime}(t) \leq \frac{\rho^{\prime}(t)}{\rho(t)} \omega_{2}(t)-\alpha \frac{R_{t_{1}}\left(\tau_{1}(t)\right)}{\rho^{1 / \alpha}(t)} \omega_{2}^{\frac{\alpha+1}{\alpha}}(t)+\rho(t) \frac{\left(r\left(\tau_{1}(t)\right)\left(z^{\prime \prime}\left(\tau_{1}(t)\right)\right)^{\alpha}\right)^{\prime}}{z^{\alpha}(t)} .
$$

By using Lemma 1, we obtain

$$
\omega_{2}^{\prime}(t) \leq \rho(t) \frac{\left(r\left(\tau_{1}(t)\right)\left(z^{\prime \prime}\left(\tau_{1}(t)\right)\right)^{\alpha}\right)^{\prime}}{z^{\alpha}(t)}+\frac{1}{(\alpha+1)^{\alpha+1}} \frac{\left(\rho_{+}^{\prime}(t)\right)^{\alpha+1}}{\left(\rho(t) R_{t_{1}}\left(\tau_{1}(t)\right)\right)^{\alpha}} .
$$

Additionally, we define another function

$$
\omega_{3}(t)=\rho(t) \frac{r\left(\tau_{2}(t)\right)\left(z^{\prime \prime}\left(\tau_{2}(t)\right)\right)^{\alpha}}{z^{\alpha}(t)} .
$$


Thus $\omega_{3}(t)>0$. Using $\left(r(t)\left(z^{\prime \prime}(t)\right)^{\alpha}\right)^{\prime}<0, \tau_{2}(t)>t$ and (14), we note that

$$
z^{\prime}(t)>\left[r\left(\tau_{2}(t)\right)\left(z^{\prime \prime}\left(\tau_{2}(t)\right)\right)^{\alpha}\right]^{1 / \alpha} R_{t_{1}}(t) .
$$

By differentiating (19) and using (20) and Lemma 1, we get

$$
\omega_{3}^{\prime}(t) \leq \rho(t) \frac{\left(r\left(\tau_{2}(t)\right)\left(z^{\prime \prime}\left(\tau_{2}(t)\right)\right)^{\alpha}\right)^{\prime}}{z^{\alpha}(t)}+\frac{1}{(\alpha+1)^{\alpha+1}} \frac{\left(\rho_{+}^{\prime}(t)\right)^{\alpha+1}}{\left(\rho(t) R_{t_{1}}(t)\right)^{\alpha}} .
$$

From (7), (15), (18) and (21), we find

$$
\begin{gathered}
\omega_{1}^{\prime}(t)+c_{1}^{\beta} \omega_{2}^{\prime}(t)+\frac{c_{2}^{\beta}}{2^{\beta-1}} \omega_{3}^{\prime}(t) \leq-\frac{\rho(t)}{4^{\beta-1}}\left(k_{1} q_{1}^{*}(t)+k_{2} q_{2}^{*}(t)\right) \frac{z^{\beta}\left(\sigma_{1}(t)\right)}{z^{\alpha}(t)} \\
+\left(1+c_{1}^{\beta}+\frac{c_{2}^{\beta}}{2^{\beta-1}}\right) \frac{1}{(\alpha+1)^{\alpha+1}} \frac{\left(\rho_{+}^{\prime}(t)\right)^{\alpha+1}}{\left(\rho(t) R_{t_{1}}^{*}(t)\right)^{\alpha}} .
\end{gathered}
$$

Using (12) and Lemma 6, we have

$$
\frac{z^{\beta}\left(\sigma_{1}(t)\right)}{z^{\alpha}(t)} \geq \frac{v^{\beta-\alpha}}{2^{\beta-\alpha} t^{2 \alpha}} \sigma_{1}^{\beta+\alpha}(t)
$$

As in the proof of Theorem 1, we are led to a contradiction with (13). This completes the proof.

In the following Theorems, we are concerned with the oscillation of solutions of Equation (1) when $\alpha=1$ and $r(t)=1$.

Theorem 3. Assume that $\left(M_{1}\right)-\left(M_{5}\right)$ and (3) hold. Let $0<\beta<1<\gamma$ and $\tau_{i}^{-1}$ exists for $i=1$, 2 . If the inequalities

$$
y^{\prime \prime \prime}(t)+\left(\frac{k_{1}}{\lambda_{1}}\right)^{\lambda_{1}}\left(\frac{k_{2}}{4^{\gamma-1} \lambda_{2}}\right)^{\lambda_{2}} \frac{\left(q_{1}^{*}(t)\right)^{\lambda_{1}}\left(q_{2}^{*}(t)\right)^{\lambda_{2}}}{\left(1+c_{1}^{\beta}+c_{2}^{\beta}\right)} y\left(\tau_{i}^{-1}\left(\sigma_{j}(t)\right)\right) \leq 0,
$$

where $i, j=1,2, i \neq j, \lambda_{1}=\frac{\gamma-1}{\gamma-\beta}$ and $\lambda_{2}=\frac{1-\beta}{\gamma-\beta}$, have oscillatory solutions, then every solution of Equation (1) is oscillatory.

Proof. Let $x$ non-oscillatory solution of Equation (1). Without loss of generality we assume that $x>0$; then, there exists a $t_{1} \geq t_{0}$ such that $x(t)>0, x\left(\tau_{i}(t)\right)>0$ and $x\left(\sigma_{i}(t)\right)>0$ for $t \geq t_{1}$ and $i=1,2$. By Lemma 6, we get that $z(t)>0, z^{\prime \prime}(t)>0$ and $z^{\prime \prime \prime}(t)<0$. Now, we define a function

$$
y(t)=z(t)+c_{1}^{\beta} z\left(\tau_{1}(t)\right)+c_{2}^{\beta} z\left(\tau_{2}(t)\right) .
$$

Thus $y(t)>0$ and $y^{\prime \prime}(t)>0$. From $(1)$ and $\left(\mathrm{M}_{5}\right)$, we obtain

$$
z^{\prime \prime \prime}(t) \leq-k_{1} q_{1}(t) x^{\beta}\left(\sigma_{1}(t)\right)-k_{2} q_{2}(t) x^{\gamma}\left(\sigma_{2}(t)\right) .
$$


Combining (24) with (25), we get

$$
\begin{aligned}
y^{\prime \prime \prime}(t)= & z^{\prime \prime \prime}(t)+c_{1}^{\beta} z^{\prime \prime \prime}\left(\tau_{1}(t)\right)+c_{2}^{\beta} z^{\prime \prime \prime}\left(\tau_{2}(t)\right) \\
\leq & -k_{1} q_{1}(t) x^{\beta}\left(\sigma_{1}(t)\right)-k_{2} q_{2}(t) x^{\gamma}\left(\sigma_{2}(t)\right) \\
& -c_{1}^{\beta}\left(-k_{1} q_{1}(t) x^{\beta}\left(\sigma_{1}\left(\tau_{1}(t)\right)\right)-k_{2} q_{2}(t) x^{\gamma}\left(\sigma_{2}\left(\tau_{1}(t)\right)\right)\right) \\
& -c_{2}^{\beta}\left(-k_{1} q_{1}(t) x^{\beta}\left(\sigma_{1}\left(\tau_{2}(t)\right)\right)-k_{2} q_{2}(t) x^{\gamma}\left(\sigma_{2}\left(\tau_{2}(t)\right)\right)\right),
\end{aligned}
$$

and so,

$$
\begin{aligned}
y^{\prime \prime \prime}(t) \leq & -k_{1} q_{1}^{*}(t)\left(x^{\beta}\left(\sigma_{1}(t)\right)+c_{1}^{\beta} x^{\beta}\left(\sigma_{1}\left(\tau_{1}(t)\right)\right)+c_{2}^{\beta} x^{\beta}\left(\sigma_{1}\left(\tau_{2}(t)\right)\right)\right) \\
& -k_{2} q_{2}^{*}(t)\left(x^{\gamma}\left(\sigma_{2}(t)\right)+c_{1}^{\beta} x^{\gamma}\left(\sigma_{2}\left(\tau_{1}(t)\right)\right)+c_{2}^{\beta} x^{\gamma}\left(\sigma_{2}\left(\tau_{2}(t)\right)\right)\right) .
\end{aligned}
$$

By Lemma 2 , since $c_{1}+c_{2}<1$ and $\beta<1<\gamma$, we obtain

$$
\begin{aligned}
y^{\prime \prime \prime}(t) & +k_{1} q_{1}^{*}(t) z^{\beta}\left(\sigma_{1}(t)\right) \\
& +k_{2} q_{2}^{*}(t)\left(x^{\gamma}\left(\sigma_{2}(t)\right)+c_{1}^{\gamma} x^{\gamma}\left(\sigma_{2}\left(\tau_{1}(t)\right)\right)+\frac{c_{2}^{\gamma}}{2^{\gamma-1}} x^{\gamma}\left(\sigma_{2}\left(\tau_{2}(t)\right)\right)\right) \leq 0 .
\end{aligned}
$$

This implies

$$
y^{\prime \prime \prime}(t)+k_{1} q_{1}^{*}(t) z^{\beta}\left(\sigma_{1}(t)\right)+\frac{k_{2}}{4^{\gamma-1}} q_{2}^{*}(t) z^{\gamma}\left(\sigma_{2}(t)\right) \leq 0 .
$$

Using Lemma 6, we have two cases for $z^{\prime}(t)$. If $z^{\prime}(t)>0$, we find

$$
y^{\prime \prime \prime}(t)+k_{1} q_{1}^{*}(t) z^{\beta}\left(\sigma_{1}(t)\right)+\frac{k_{2}}{4^{\gamma-1}} q_{2}^{*}(t) z^{\gamma}\left(\sigma_{1}(t)\right) \leq 0 .
$$

Using arithmetic-geometric mean inequality with $u_{1}=\frac{k_{1}}{\lambda_{1}} q_{1}^{*}(t) z^{\beta}\left(\sigma_{1}(t)\right)$ and $u_{2}=$ $\frac{k_{2}}{4^{\gamma-1} \lambda_{2}} q_{2}^{*}(t) z^{\gamma}\left(\sigma_{1}(t)\right)$, we get

$$
\begin{aligned}
\lambda_{1} u_{1}+\lambda_{2} u_{2} & \geq u_{1}^{\lambda_{1}} u_{2}^{\lambda_{2}} \\
& =\left(\frac{k_{1}}{\lambda_{1}}\right)^{\lambda_{1}}\left(\frac{k_{2}}{4^{\gamma-1} \lambda_{2}}\right)^{\lambda_{2}}\left(q_{1}^{*}(t)\right)^{\lambda_{1}}\left(q_{2}^{*}(t)\right)^{\lambda_{2}} z\left(\sigma_{1}(t)\right) .
\end{aligned}
$$

Since $\tau_{1}(t)<t<\tau_{2}(t)$, we note that

$$
y(t) \leq\left(1+c_{1}^{\beta}+c_{2}^{\beta}\right) z\left(\tau_{2}(t)\right) .
$$

Hence, from (28), (27) becomes

$$
y^{\prime \prime \prime}(t)+\left(\frac{k_{1}}{\lambda_{1}}\right)^{\lambda_{1}}\left(\frac{k_{2}}{4^{\gamma-1} \lambda_{2}}\right)^{\lambda_{2}} \frac{\left(q_{1}^{*}(t)\right)^{\lambda_{1}}\left(q_{2}^{*}(t)\right)^{\lambda_{2}}}{\left(1+c_{1}^{\beta}+c_{2}^{\beta}\right)} y\left(\tau_{2}^{-1}\left(\sigma_{1}(t)\right)\right) \leq 0 .
$$

Then, the condition (23) implies (29) has oscillatory solution, which contradicts $y(t)>0$. 
Let $z^{\prime}(t)<0$. As in the previous case, we get

$$
y^{\prime \prime \prime}(t)+\left(\frac{k_{1}}{\lambda_{1}}\right)^{\lambda_{1}}\left(\frac{k_{2}}{4^{\gamma-1} \lambda_{2}}\right)^{\lambda_{2}} \frac{\left(q_{1}^{*}(t)\right)^{\lambda_{1}}\left(q_{2}^{*}(t)\right)^{\lambda_{2}}}{\left(1+c_{1}^{\beta}+c_{2}^{\beta}\right)} y\left(\tau_{1}^{-1}\left(\sigma_{2}(t)\right)\right) \leq 0 .
$$

Hence, the condition (23) implies (30) has oscillatory solution, which contradicts $y(t)>0$. This contradiction completes the proof.

Remark 2. There are numerous results concerning the oscillation of the equation

$$
y^{\prime \prime \prime}(t)+q(t) y(\sigma(t))=0,
$$

(see $[2,18,20,21])$, which include Hille and Nehari types, Philos type, etc.

Assume that

$$
\tau_{i}(t)=t+(-1)^{i} \widetilde{\tau}_{i}, \sigma_{i}(t)=t-(-1)^{i} \widetilde{\sigma}_{i}
$$

where $\widetilde{\tau}_{i}, \widetilde{\sigma}_{i}$ are positive constants for $i=1$, 2. It is well known (see [9]) that the differential inequalities (29) and (30) are oscillatory if

$$
\liminf _{t \rightarrow \infty} \int_{t-\left(\widetilde{\tau}_{2}+\widetilde{\sigma}_{1}\right) / 3}^{t}\left(\widetilde{\tau}_{2}+\widetilde{\sigma}_{1}\right)^{2}\left(q_{1}^{*}(t)\right)^{\lambda_{1}}\left(q_{2}^{*}(t)\right)^{\lambda_{2}}>\frac{9}{2 e}\left(\frac{\lambda_{1}}{k_{1}}\right)^{\lambda_{1}}\left(\frac{4^{\gamma-1} \lambda_{2}}{k_{2}}\right)^{\lambda_{2}}
$$

and

$$
\liminf _{t \rightarrow \infty} \int_{t}^{t+\widetilde{\tau}_{1}+\widetilde{\sigma}_{2}}(s-t)^{2}\left(q_{1}^{*}(t)\right)^{\lambda_{1}}\left(q_{2}^{*}(t)\right)^{\lambda_{2}}>2\left(\frac{\lambda_{1}}{k_{1}}\right)^{\lambda_{1}}\left(\frac{4^{\gamma-1} \lambda_{2}}{k_{2}}\right)^{\lambda_{2}}
$$

respectively. Hence, we conclude the following theorem:

Theorem 4. Assume that $0<\beta<1<\gamma$ and (31) hold. If (32) and (33) hold, then every solution of Equation (1) is oscillatory.

Remark 3. In the case where $\alpha=1, r(t)=1$ and $p_{i}(t)=0$, Equation (1) becomes

$$
x^{\prime \prime \prime}(t)+q_{1}(t) f_{1}\left(x\left(\sigma_{1}(t)\right)\right)+q_{2}(t) f_{2}\left(x\left(\sigma_{2}(t)\right)\right)=0 .
$$

Baculikova and Dzurina [5] proved that every nonoscillatory solution $x$ of (34) satisfies $x^{\prime}<0$. Thus, Theorems 3 and 4 improve the results in [5].

Remark 4. A manner similar to the Theorem 3, we can study the oscillation of solutions of Equation (1) when $0<\gamma<1<\beta$.

Remark 5. If $\alpha=1, f_{1}(x)=x^{\beta}, f_{2}(x)=x^{\gamma}, \tau_{1}(t)=t-\widetilde{\tau}_{1}, \sigma_{1}(t)=t-\widetilde{\sigma}_{1}, \tau_{2}(t)=t+\widetilde{\tau}_{2}, \sigma_{2}(t)=t+\widetilde{\sigma}_{2}$ and $\widetilde{\tau}_{i}, \widetilde{\sigma}_{i}$ are positive constants, then Theorem 1 extends Theorem 2.5 and 2.7 in [23].

Remark 6. The results of Theorem 3 can be extended to the third-order differential equation

$$
\left((z(t))^{\alpha}\right)^{\prime \prime \prime}+q_{1}(t) f_{1}\left(x\left(\sigma_{1}(t)\right)\right)+q_{2}(t) f_{2}\left(x\left(\sigma_{2}(t)\right)\right)=0 ;
$$


the details are left to the reader.

Example 1. Consider the equation

$$
\left(x+\frac{1}{3} x\left(\frac{1}{3} t\right)+\frac{1}{3} x(2 t)\right)^{\prime \prime \prime}+\frac{q_{0}}{t^{3}} x\left(\frac{1}{2} t\right)+\frac{q_{1}}{t^{3}} x(2 t)=0,
$$

where $q_{0}>0$. We note that $\alpha=\beta=\gamma=1, r(t)=1, p_{1}(t)=p_{2}(t)=1 / 3, \tau_{1}(t)=1 / 3 t, \sigma_{1}(t)=1 / 2 t$, $\tau_{2}(t)=\sigma_{2}(t)=2 / t$ and $q^{*}(t)=q_{0} / t^{3}$. Hence, it is easy to see that

$$
\int_{t_{0}}^{\infty} \frac{1}{r^{1 / \alpha}(s)} \mathrm{d} s=\infty
$$

Now, if we set $\rho(s):=t$ and $k_{1}=k_{2}=1$, then we have

$$
\Theta_{1}(t)=\frac{q_{0}}{2 s} .
$$

Thus, we find

$$
\begin{aligned}
& \limsup _{t \rightarrow \infty} \int_{t_{0}}^{t}\left(\Theta_{1}(s)-\left(1+c_{1}^{\beta}+\frac{c_{2}^{\beta}}{2^{\beta-1}}\right) \frac{1}{(\alpha+1)^{\alpha+1}} \frac{\left(\rho_{+}^{\prime}(s)\right)^{\alpha+1} r\left(\sigma_{1}(s)\right)}{\left(\rho(s) \sigma_{1}^{\prime}(s)\right)^{\alpha}}\right) d s \\
= & \limsup _{t \rightarrow \infty} \int_{t_{0}}^{t}\left(\frac{q_{0}}{2 s}-\frac{5}{6 s}\right) d s .
\end{aligned}
$$

Thus, the conditions become

$$
q_{0}>1.66
$$

Thus, by using Theorem 1, Equation (35) is either oscillatory if $q_{0}>1.66$ or tends to zero as $t \rightarrow \infty$.

Author Contributions: O.M. and O.B.: Writing original draft, and writing review and editing. D.C.: Formal analysis, writing review and editing, funding and supervision. All authors have read and agreed to the published version of the manuscript.

Funding: The authors received no direct funding for this work.

Acknowledgments: The authors thank the reviewers for for their useful comments, which led to the improvement of the content of the paper.

Conflicts of Interest: There are no competing interests for the authors.

\section{References}

1. Hale, J.K. Theory of Functional Differential Equations; Springer: New York, NY, USA, 1977.

2. Agarwal, R.P.; Bohner, M.; Li, T.; Zhang, C. Oscillation of third-order nonlinear delay differential equations. Taiwanese J. Math. 2013, 17, 545-558. [CrossRef]

3. Baculikova, B.; Dzurina, J. On the asymptotic behavior of a class of third order nonlinear neutral differential equations. Cent. Eur. J. Math. 2010, 8, 1091-1103. [CrossRef]

4. Baculikova, B.; Dzurina, J. Oscillation of third-order neutral differential equations. Math. Comput. Modell. 2010, 52, 215-226. [CrossRef]

5. Baculikova, B.; Dzurina, J. Some Properties of Third-Order Differential Equations with Mixed Arguments. J. Math. 2013, 2013, 528279. [CrossRef] 
6. Bazighifan, O.; Cesarano, C. Some New Oscillation Criteria for Second-Order Neutral Differential Equations with Delayed Arguments. Mathematics 2019, 7, 619. [CrossRef]

7. Bazighifan, O.; Elabbasy, E.M.; Moaaz, O. Oscillation of higher-order differential equations with distributed delay. J. Inequal. Appl. 2019, 55, 1-9. [CrossRef]

8. Chatzarakis, G.E.; Elabbasy, E.M.; Bazighifan, O. An oscillation criterion in 4th-order neutral differential equations with a continuously distributed delay. Adv. Differ. Equ. 2019, 336, 1-9.

9. Das, P. Oscillation criteria for odd order neutral equations. J. Math. Anal. Appl. 1994, 188, 245-257. [CrossRef]

10. Elabbasy, E.M.; Hassan, T.S.; Moaaz, O. Oscillation behavior of second-order nonlinear neutral differential equations with deviating arguments. Opusc. Math. 2012, 32, 719-730. [CrossRef]

11. Elabbasy, E.M.; Moaaz, O. On the asymptotic behavior of third-order nonlinear functional differential equations. Serdica Math. J. 2016, 42, 157-174.

12. Elabbasy, E.M.; Barsoum, M.Y.; Moaaz, O. Boundedness and oscillation of third order neutral differential equations with deviating arguments. J. Appl. Math. Phys. 2015, 3, 1367-1375. [CrossRef]

13. Elabbasy, E.M.; Moaaz, O. On the oscillation of third order neutral differential equations. Asian J. Math. Appl. 2016, 2016, 0274.

14. Elabbasy, E.M.; Moaaz, O.; Almehabresh, E.S. Oscillation Properties of Third Order Neutral Delay Differential Equations. Appl. Math. 2016, 7, 1780-1788. [CrossRef]

15. Bazighifan, O.; Cesarano, C. A Philos-Type Oscillation Criteria for Fourth-Order Neutral Differential Equations. Symmetry 2020, 12, 379. [CrossRef]

16. Elabbasy, E.M.; Moaaz, O. Oscillation Criteria for third order nonlinear neutral differential equations with deviating arguments. Int. J. Sci. Res. 2016, 5, 1.

17. Kiguradze, I.T.; Chanturia, T.A. Asymptotic Properties of Solutions of Nonautonomous Ordinary Differential Equations; Kluwer Academic Publishers: Drodrcht, The Netherlands, 1993.

18. Ladas, G.; Sficas, Y.G.; Stavroulakis, I.P. Necessary and sufficent conditions for oscillation of higher order delay differential equations. Trans. Am. Math. Soc. 1984, 285, 81-90. [CrossRef]

19. Moaaz, O.; Awrejcewicz, J.; Bazighifan, O. A New Approach in the Study of Oscillation Criteria of Even-Order Neutral Differential Equations. Mathematics 2020, 8, 197. [CrossRef]

20. Saker, S.H.; Dzurina, J. On the oscillation of certain class of third-order nonlinear delay differential equations. Math. Bohemica 2010, 135, 225-237.

21. Saker, S.H. Oscillation criteria of Hille and Nehari types for third-order delay differential equations. Comm. Appl. Anal. 2007, 11, 451-468.

22. Han, Z.; Li, T.; Zhang, C.; Sun, S. Oscillatory Behavior of Solutions of Certain Third-Order Mixed Neutral Functional Differential Equations. Bull. Malays. Math. Sci. Soc. 2012, 35, 611-620.

23. Thandapani, E.; Rama, R. Oscillatory behavior of solutions of certain third order mixed neutral differential equations. Tamkang J. Math. 2013, 44, 99-112. [CrossRef]

24. Thandapani, E.; Li, T. On the oscillation of third-order quasi-linear neutral functional differential equations. Arch. Math. 2011, 47, 181-199.

(C) 2020 by the authors. Licensee MDPI, Basel, Switzerland. This article is an open access article distributed under the terms and conditions of the Creative Commons Attribution (CC BY) license (http:/ / creativecommons.org/licenses/by/4.0/). 